\title{
FINITE ELEMENT ANALYSIS OF DIFFUSION PROCESSES IN WHITE DWARFS
}

\author{
C. Pelletier, G. Fontaine, and F. Wesemael
}

Département de Physique, Université de Montréal

The spectral evolution of white dwarfs is governed by diffusion processes which enter into competition with mechanisms such as mass loss, convective mixing, and accretion from the interstellar medium in various phases of the evolution. Until recently, our theoretical understanding of the chemical evolution of these stars has been limited by the very severe numerical difficulties which plague a time-dependent description of the problem. Indeed, diffusion problems in white dwarf interiors and envelopes are particularly demanding from a computational standpoint: they involve relative chemical abundances spanning many orders of magnitude, time integration length of a few billion years, and many physical processes operating with greatly different time constants. We have already introduced in the field a robust numerical technique based on an implicit finite difference scheme designed for nonlinear two-point boundary value problems (Pelletier 1986). This method has been used to investigate a number of problems related to the spectral evolution of white dwarfs (Pelletier 1986; Pelletier et al. 1986; Dupuis et al. 1987). As requirements for further progress in the field become more exacting and in the interest of improving the efficiency, we have sought to develop even more powerful numerical techniques. We briefly introduce here an efficient computational approach to diffusion problems in white dwarfs based on a Galerkin finite element method to solve the convective-diffusion equation in an evolving white dwarf model. As an illustrative example, we discuss some sample results of a detailed investigation of the problem of chemical sedimentation $(H, H e$, and $C$ ) in the envelopes of hot white dwarfs and the formation of DA stars.

The convective-diffusion problems encountered in simulating white dwarf evolution are governed by a general nonlinear parabolic equation of the form

$$
\frac{\partial u}{\partial t}=\frac{\partial}{\partial r}\left(D(r, u) \frac{\partial u}{\partial r}\right)+F\left(r, u, \frac{\partial u}{\partial r}\right)
$$

where $u$ is a normalized relative abundance, $D$ and $F$ are general nonlinear functions containing information about the diffusion properties, the model's physical parameters, geometry and external forces, and $(r, t)$ are the radius and time. The fundamental concept of a finite element algorithm is the assumption of known functional dependence for $u(r, t)$ on disjoint, contiguous subdomains of the integration interval; these subdomains are termed finite elements. The first task is to expand the dependent variable $u$ in terms of a set of basis functions $\left\{\phi_{j}^{(e)}(r)\right\}$, each of which is a low-order polynomial (usually a Lagrange interpolating polynomial) of compact support (i.e. $\phi_{j}^{(e)}$ is non-zero over only one element). We assume that $u$ is adequately interpolated on each element by a truncated power series of the form

$$
\tilde{u}(r, t)=\sum_{j=1}^{l} c_{j}(t) \phi_{j}^{(e)}(r)
$$

where $\left\{c_{j}(t)\right\}$ is the set of unknown coefficients of the basis functions and $l-1$ is the degree of the polynomial basis; the interest is thus shifted toward the coefficients $c_{j}(t)$ which completely specify the solution. We next 
insert these expansions into (1) and form what is called a residual:

$$
R(r ; c)=\frac{\partial \tilde{u}}{\partial t}-\frac{\partial}{\partial r}\left(D(r, \tilde{u}) \frac{\partial \tilde{u}}{\partial r}\right)-F\left(r, \tilde{u}, \frac{\partial \tilde{u}}{\partial r}\right)
$$

The residual is a representation of the numerical error introduced on each element by the power series (2); the Galerkin formulation aims at minimizing (3) by orthogonalizing the error to the basis functions. We thus impose for each element:

$$
\int_{e} R(r ; c) \phi_{j}^{(e)}(r) d r=0 \quad j=1, \ldots, N
$$

( $N=$ number of elements). This process yields for the $c_{j}(t)$ a system of first-order ordinary differential equations (ODE) of the form:

$$
[K]\{C\}^{\prime}+[L]\{C\}+\{M\}=\{0\}
$$

where the coefficients of matrix $[K]$ are given by the orthogonalization properties of the polynomial basis, the coefficients of $[L]$ are related to integrals of function $D$ and the vector $\{M\}$ contains integrals of $F$ and information about the boundary conditions (the superscript prime denotes differentiation with respect to time). Using the finite element procedure transforms an initial-boundary-value problem in a pure initial-value problem. With finite difference theory, the $\mathrm{K}$ matrix in equation (5) would be defined as the identity matrix. Finite element theory predicts that $[K]$ be non-diagonal with bandwidth a function of discretization and the degree of the polynomial basis.

If $P$ denotes the degree of the polynomial basis ( $P=3$ in our simulations), then equation (5) is made of $N P+1$ highly non-linear stiff ODE (an ODE system is called stiff if it has both very rapidly/slowly changing components; a white dwarf is prone to this situation since the diffusion time scales widely differ from one end of the integration domain to the other). The primary difficulty with a stiff system is that most conventional methods for solving it require unrealistic small values of the time step and the solution either blows up or cannot be computed in an affordable CPU time. Even if one can bear the expense, classical methods of solution require so many steps that roundoff errors invalidate the solution. To perform the complete computation, we must use specially designed techniques for stiff systems; we adopted the famous Gear backward differentiation formulas modified to take into account the special structure of (5) induced by the finite element formulation (c.f. Gear 1971 and Hindmarsh 1977).

We are currently using this finite element technique in an ungoing detailed investigation of the problem of element sedimentation in the envelopes of hot white dwarfs. The basic premise is the idea that PG 1159-type stars could be the progenitors of the majority of the white dwarfs (Fontaine and Wesemael 1987). In this scenario, it is envisioned that very small traces of $H$ (the tail of the original distribution) are left in the envelope of a pre-white dwarf after the final phase of significant mass loss has terminated. The expected structure of such an object is that of a $\mathrm{C} / \mathrm{O}$ core surrounded by a $\mathrm{He}$-rich mantle and a $\mathrm{He}$-dominated atmosphere. With time, hydrogen diffuses to the surface and, when an atmosphere worth of this element has accumulated, a newborn DA star can be observed.

In our time-dependent simulations of such events, we have idealized the situation by considering an initial discontinuous distribution made of an almost pure $C$ core surrounded by an almost pure $H e$ layer; this mimics very roughly the sharp front due to past $\mathrm{He}$-burning reactions. 


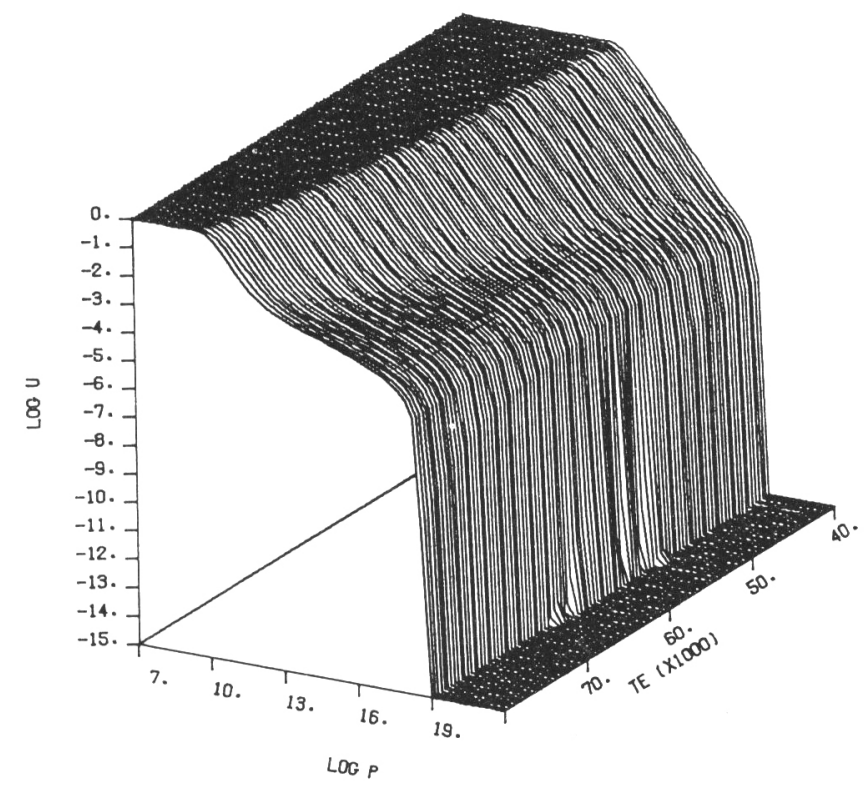

Figure 1. Evolution of the hydrogen distribution in a $0.6 M_{\odot}$ model. The star almost instantly becomes a DA, having at $80,000 \mathrm{~K}$ a hydrogen layer of $\log q(H) \approx-11.7$; at $40,000 \mathrm{~K}$, $\log q(H) \approx-8.7$.

For simplicity, the small traces of $H$ in the envelope are assumed to be initially uniform. Diffusion alters very quickly these initial configurations. Thus, the evolution of the $H$ distribution is followed in presence of residual $H$ burning and in presence of a changing background due to the diffusion of $H e$ and $C$ in the model. Fig. 1 illustrates a sample result for the evolution of a $0.6 M_{\odot}$ model with a relatively large quantity of $H$. The figure shows how the $H$ profile $(\log u \equiv \log \{n(H) /[n(H)+n(H e)+n(C)]\})$ given in terms of the pressure $(\log P)$ evolves with decreasing effective temperature. In this particular sequence, the assumed quantity of $H$ is so large ( $\log u=-4.0$ uniformly distributed in the initial phases), that $H$ accumulates very quickly at the surface and the model turns into a DA white dwarf in about 225 years. These rapid phases are not shown in the figure, and the first profile illustrated corresponds to an age of a $80,000 \mathrm{~K}$ star; already a significant layer of $H$ (much more than the thickness of an atmosphere) has built up. The presence of residual nuclear burning is quite obvious as it creates a sharp quasi-stationary front. The hydrogen located above that front is submitted to the concentration gradient's downward pull and the upward push of the pressure gradient. As can be seen, the natural tendency of hydrogen to "float" clearly wins and the top layer simply thickens with time. Nuclear burning slows down the general migration but cannot prevent the transformation of the star into a DA object. The separation is completed when the profile reaches a quasi-equilibrium configuration around $T_{e} \simeq 40,000 \mathrm{~K}$. This particular computation required some 6664 time steps with a moving grid made of 120 elements.

Convective-diffusion simulations in white dwarfs are large numerical problems, so efficiency is important. The Galerkin finite element formulation presented here performed very satisfactorily, with increased efficiency when 
compared to finite difference codes. For instance, the new code has on the average completed a typical run 5 times faster then the old one used by Pelletier et al. (1986) with no significant differences in the results. The overall performance of the finite element method has been quite impressive for all the simulations that we attempted (for further applications, see the paper by Dupuis et al. 1988). The resulting algorithm is accurate, fast and stable even for numerically "explosive" problems involving hydrogen.

This research has been supported by the NSERC Canada and a E.W.R. Steacie Fellowship to one of us (GF).

\section{References}

Dupuis, J., Pelletier, C., Fontaine, G., and Wesemael, F. 1988, these proceedings.

Dupuis, J., Pelletier, C., Fontaine, G., and Wesemael, F. 1987, in IAU Colloquium 95, The Second Conference on Faint Blue Stars, eds. A.G.D. Philip, D.S. Hayes, and J. Liebert (Schenectady: L. Davis Press), p. 657.

Fontaine, G., and Wesemael, F. 1987, in IAU Colloquium 95, The Second Conference on Faint Blue Stars, eds. A.G.D Philip, D.S. Hayes, and J. Liebert (Schenectady: L. Davis Press), p. 319.

Gear, C. W. 1971, Numerical Initial Value Problems in Ordinary Differential Equations (Prentice-Hall).

Hindmarsh, A. C. 1977, GEARB: Solution of Ondinary Differential Equations Having Banded Jacobian, Lawrence Livermore Lab. Rep. UCID-30059.

Pelletier, C. 1986, Ph.D. thesis, Université de Montréal.

Pelletier, C., Fontaine, G., Wesemael, F., Michaud, G., and Wegner, G. 1986, Astrophys. J., 307, 242. 